Anuario Latinoamericano Ciencias Políticas

y Relaciones Internacionales

vol. 8, 2019

pp. $37-48$

\section{El desarrollo de las perspectivas feministas en el estudio disciplinar de las Relaciones Internacionales en el mundo y en Argentina}

\author{
The Development of Feminist Perspectives \\ in the Disciplinary Study of International Relations \\ in the World and in Argentina
}

\author{
Mariel R. Lucero* \\ FACULTAD DE CIENCIAS POLÍTICAS Y SOCIALES \\ UNIVERSIDAD NACIONAL DE CUYO, ARGENTINA \\ $\triangle$ mlucero@fcp.uncu.edu.ar \\ https://orcid.org/0000-0002-9904-5238
}

DOI: $10.17951 /$ al.2019.8.37-48

\title{
RESUMEN
}

Este trabajo presenta un estado de la cuestión sobre las perspectivas feministas en Relaciones Internacionales a nivel global, donde aborda sucintamente el posicionamiento epistemológico y metodológico del feminismo, para desarrollar la génesis y características de los principales enfoques feministas en las Relaciones Internacionales y sus objetivos; y en una segunda instancia, revela el estado de los estudios en Argentina en particular.

PALABRAS CLAVE: Relaciones Internacionales, enfoque feminista, mujeres, Latinoamérica.

\footnotetext{
ABSTRACT

This work presents a state of the art about feminist perspectives in International Relations at a global level, where it briefly addresses epistemological and methodological positioning of feminism to develop the genesis and characteristics of the main feminist approaches in International Relations and their objectives; and in a second section, it reveals the state of the studies in Argentina in particular.

KEYWORDS: International Relations, feminist approach, women, Latin America.

* Doctoranda en Relaciones Internacionales por la UNLP. Magister en Relaciones Internacionales por FLACSO/Argentina. Directora del Centro de Estudios en Relaciones Internacionales y Medio Ambiente (CERIMA-UNCUyo). Profesora en la FCPyS-UNCUyo. Especialista en mujeres y diplomacia, mujeres y fuerzas armadas, enfoques feministas en Relaciones Internacionales.
} 

Dossier
América Latina:
género y política

\section{Introducción}

Este trabajo aborda sucintamente la génesis y características de los principales enfoques feministas en las Relaciones Internacionales y sus objetivos, explicando su posicionamiento epistemológico y metodológico, mostrando el panorama de la cuestión a nivel global; y en una segunda parte, presentando el estado de los estudios en Argentina en particular.

Ello nos obliga a remontarnos al origen de estos enfoques y los primeros aportes teóricos dentro de la disciplina a fines de la década de los ochenta del siglo pasado. Sin embargo, como sostienen J. Ann Tickner y Jaqui True, no es el feminismo el que llega tarde a las Relaciones Internacionales sino las Relaciones Internacionales llegan tarde al feminismo, principalmente si tenemos presente que durante el período de nacimiento de la disciplina -entreguerrasla actividad pacifista estuvo intensamente impulsada con los aportes que diversas mujeres hicieron a esta corriente (2018, p. 29).

En este sentido, para comenzar el estudio es necesario considerar la praxis feminista en el relato, lo cual nos lleva inexorablemente a trasladarnos al contexto de desarrollo del movimiento feminista que fue el motor de la productividad en el campo académico.

\section{Aparición de los enfoques feministas dentro de la disciplina}

La primera ola del feminismo se inició con la lucha en defensa de los derechos políticos y laborales de las mujeres a comienzos del siglo XX, y tuvo una etapa posterior -el "nuevo feminismo" o revolución feminista- en la década de los setenta. Este segundo período se asocia principalmente a los slogans: "lo personal es político" y "no se nace mujer, se hace", que son indicativos del profundo replanteo estructural que presentaban, encontrando como una de sus exponentes más icónicas a Simone de Beauvoir. Este generó no sólo acción en las calles, sino también un productivo pensamiento reflexivo dentro de la academia $^{1}$. La mirada epistemológica del feminismo cuestionó y cuestiona las producciones científicas tradicionales que sostienen la universalidad, la neutralidad y la objetividad de la ciencia, planteando que ésta es el resultado parcial de una representación del orden simbólico, social y político patriarcal de un momento determinado.

Al respecto, la filósofa y epistemóloga norteamericana Sandra Harding destaca que la ciencia se encuentra permeada por la moral y la política, lo cual

\footnotetext{
1 Cuando hablamos de pensamiento académico nos referimos a la producción de un pensamiento feminista científico, constante, aportado desde mujeres y varones, a diferencia de otras épocas donde los aportes de mujeres a la academia provenían desde el exterior, ya que muchas tenían vedada su participación en este ámbito debido a razones "culturales patriarcales".
} 
le genera cierto carácter “poroso”. Allí ambas inciden en la estructuración del esquema conceptual y metodológico científico en un determinado momento histórico. Mientras tanto, el discurso tradicional mantiene su imagen de "peligrosa" neutralidad -en un sentido social y epistemológico- mostrando una interpretación exclusiva y única de la realidad, escondida bajo un manto de objetividad. Por tanto, según Harding, la relación entre la ciencia y la política debe ser reinterpretada, entendiendo cada una de estas partes como un todo, sin desvincular el contexto de producción. Al respecto, Michel Foucault reafirma la mirada sobre esta interrelación sosteniendo que "el ejercicio del poder crea perpetuamente conocimiento y, a la inversa, el conocimiento constantemente induce efectos de poder [...] no es posible para el poder ser ejercido sin conocimiento, es imposible para el conocimiento no engendrar poder" 2 (Wibben, 2004, p. 102).

En este sentido, las teorías feministas adoptan un posicionamiento científico crítico, rechazando el enfoque monolítico positivista. Jill Steans, entre otras especialistas, sostiene que lo esencial en el acercamiento al estudio del pensamiento feminista de las Relaciones Internacionales es la diversidad (Peterson, 2004, p. 36 y 271; Sylvester, 1992, pp. 32-35; Steans, 2006, pp. 6-10). Dentro del campo disciplinar, el feminismo en sus diversas vertientes se ubica en el cuarto debate teórico dentro de la corriente reflectivista. Esto le permite acomodarse dentro de una visión pluriparadigmática, en oposición a la idea de desarrollo de la ciencia tradicional en torno a un único paradigma, sostenida por Thomas Kuhn, y representada por los racionalistas y su enfoque teórico más significativo: el realismo (Sodupe, 2003, pp. 46-48).

La ubicación del feminismo dentro del cuarto debate en las Relaciones Internacionales refiere a una discusión entre el pensamiento predominante en la disciplina ${ }^{3}$, sostenido por el enfoque racionalista -compuesto por realistas, neorrealistas e institucionalistas liberales- frente a los "reflectivistas". Al interior de esta corriente confluyen seis perspectivas: la constructivista, la escuela inglesa, la posmodernista, la posestructuralista, las teorías críticas y las feministas. Kepa Sodupe argumenta que el reflectivismo se construyó "haciendo hincapié en (la) reflexividad teórica [...] el interés por reflexionar sobre el propio proceso de teorización” (Sodupe, 2003, p. 19). Desde esta posi-

\footnotetext{
2 Traducción de la autora.

3 Kepa Sodupe se diferencia de otros autores como S. Hoffmann, Maghroori, Dougherty y Pfaltzgraff, que sostienen el final de las discusiones en Relaciones Internacionales en el tercer debate. Sodupe establece como primer debate al existente entre idealistas y realistas que se desarrolló entre las décadas de los 20 a los 50, junto con el nacimiento de la disciplina. Al segundo debate, entre tradicionalistas y behavioristas o cientificistas, entre las décadas de los 50 hasta fines de los 60. Al tercer debate, o debate interparadigmático, lo denomina a aquel donde la disciplina deja de centrarse en el paradigma tradicional estatocéntrico para incorporar nuevos paradigmas: el globalista y el sistémico, discusión que ocupó las décadas de los 70 a los 80; y finalmente, el cuarto debate centrado en una discusión epistemológica, metodológica y ontológica que enfrenta a racionalistas y reflectivistas. (Sodupe, 2003, pp. 16-20 y pp. 52-62).
}

El desarrollo de las perspectivas feministas en el estudio disciplinar de las Relaciones Internacionales en el mundo y en Argentina

Mariel R. Lucero 
Dossier América Latina: género y política ción cuestionan desde lo epistemológico, lo metodológico y lo ontológico a la corriente racionalista.

El reflectivismo propone una interpretación pluralista de enfoques, fomentando el debate, entendiendo que la multiplicidad de interpretaciones en el diálogo es lo que genera avances en el desarrollo científico de la disciplina. En oposición a los enfoques racionalistas, se diferencia en tres planos: el ontológico, el epistemológico y el metodológico. El ontológico se centra en una visión holista, que entiende que las estructuras son primordialmente moldeadas por las ideas antes que por las fuerzas materiales y que, al mismo tiempo, son "constructoras de la identidad e intereses de los agentes", entendiendo a las estructuras como condicionantes del comportamiento de los agentes, con una visión de la sociedad construida primordialmente desde arriba hacia abajo, pero en un proceso de retroalimentación.

Por otra parte, en lo epistemológico y metodológico, el objeto de estudio se centra en los hechos sociales y en base a ello privilegia la comprensión, a diferencia del racionalismo que pondera la explicación y la perspectiva propia de las ciencias naturales. En este sentido, los reflectivistas promueven la pluralidad de interpretaciones, en particular destacan la importancia del contexto histórico en el cual se produce el conocimiento. Entienden que "si para interpretar el sentido de las acciones humanas éstas han de insertarse en el conjunto de los valores y las prácticas sociales imperantes en el momento histórico en que ocurrieron, entonces los conceptos de verdad y conocimiento se hallan entroncados en la historia y no por encima de ella" (Sodupe, 2003, p. 69). Por ello, cualquier interpretación puede ser válida si respeta los criterios científicos convencionales propios de cada disciplina, siendo algunos de los métodos más empleados por los reflectivistas, por ejemplo, el etnográfico y la hermenéutica. En oposición, en el racionalismo sólo la falsación y la verificación son los determinantes del conocimiento científico. En otras palabras, para ellos sólo existe conocimiento científico cuando es empíricamente contrastable. En este marco reflectivista se encuadra el feminismo en las Relaciones Internacionales.

A fines de los años ochenta, el contexto histórico mundial con la caída del muro de Berlín y el fracaso del paradigma realista para proyectar dicho desenlace favorecieron el surgimiento de aportes críticos, abriendo espacio a nuevos enfoques para analizar la política frente a un "nuevo" orden internacional. Esto, sumado al empuje de la oleada feminista, favoreció su ingreso en las Relaciones Internacionales, uno de los últimos campos científicos, debido a que es un ámbito de "discurso patriarcal crudo", como sostiene R. B. J. Walker (Jarvis, 2000, p. 145). En estos inicios encontramos, por un lado, el impulso dado por las publicaciones consideradas pilares y que podemos nuclear en una tríada clásica: el libro de Jean Bethke Elshtain Women and War de 1987; Bananas, Beaches and Bases. Making Feminist Sense in International Relations de Cinthia Enloe, de 1990, y Gender in International Relations de J. Ann Tickner, de 1992. En particular el libro de Enloe refiere a hacer presente el feminismo en las Relaciones Internacionales entendiendo esto no como un 
mero reciclaje sino reflexionando sobre cómo se produce el conocimiento y se seleccionan los temas de la política internacional desde otro enfoque, cuestionando las estructuras tradicionales patriarco-estatales.

En este sentido, el impulso se reforzó con una serie de conferencias. En el Reino Unido se organizó un encuentro en 1988, a través de la London School of Economics, y en los Estados Unidos, en los años siguientes, en la Universidad del Sur de California, y en 1990 en Wellesley College. Estos encuentros funcionaron como disparadores de un debate académico prolífero, necesario para el ingreso definitivo del enfoque feminista, que se reflejaría en el lanzamiento de un número especial, en 1988, dedicado a "Mujeres y Relaciones Internacionales" en la revista Millennium. Journal of International Studies, y en 1990, en International Studies Quarterly. Finalmente, en 1992, la aparición de paneles sobre género en el Congreso de International Studies Association (ISA), y la creación de un grupo de estudios feministas dentro de la misma asociación llamada "Estudios de género y teoría feminista" (Feminist Theory and Gender Studies), al igual que su par en el Reino Unido, British International Studies Association (BISA), marcarían la instalación definitiva de esta perspectiva dentro de la disciplina (Wibben, 2004, pp. 98-99; Sylvester, 2004, pp.9-15; Steans, 2006, pp. 1-2). Este contexto fue generador de numerosas publicaciones y cursos sobre género en las Relaciones Internacionales en los programas de estudio anglosajones en los años subsiguientes.

La particularidad de la corriente feminista es que incorpora y visibiliza a las mujeres dentro de las Relaciones Internacionales como actoras ${ }^{4}$ del sistema internacional, estudiando su interrelación con otras y otros actores. Esta perspectiva aborda las relaciones de poder existentes entre varones y mujeres desde el ámbito privado hacia el plano internacional y viceversa, entendiendo que es un proceso de retroalimentación permanente que replica las relaciones de dominación que provocan subordinación y discriminación hacia las mujeres. Por ello, resulta esencial para esta concepción superar la separación entre lo público y lo privado. Sin embargo, como ya se mencionó, la perspectiva feminista carece de unicidad, por ello resulta interesante caracterizar las distintas corrientes existentes dentro de ella.

\section{Las diversas perspectivas feministas dentro de las Relaciones Internacionales}

Antes de comenzar con el análisis de las distintas corrientes es necesario aclarar que, como campo construido interdisciplinarmente, muchos de los aportes, en este caso de conceptos y categorías feministas, son externos a la disci-

\footnotetext{
4 Se utiliza el vocablo actoras para diferenciarlo de actrices, ya que este último se relaciona con la actuación artística y la falsedad, y lo que nos interesa es destacar a las mujeres como sujetas sociales.
}

El desarrollo de las perspectivas feministas en el estudio disciplinar de las Relaciones Internacionales en el mundo y en Argentina

Mariel R. Lucero 
Dossier América Latina: género y política plina, y terminan siendo centrales en el desarrollo y comprensión del análisis de la perspectiva feminista dentro de las Relaciones Internacionales. Por ello, consideramos indispensable introducir al menos dos de ellos, aunque su abordaje sea sucinto, sin profundizar en el debate teórico que estos generan dentro del feminismo. Por otro lado, resulta significativo destacar que algunos de los trabajos no surgen específicamente de la disciplina, sin embargo, contribuyeron y contribuyen a su desarrollo y por ello sus autores y autoras son considerados como referentes.

A comienzos de la década de los setenta, de la mano de Kate Millet aparece la "teoría del patriarcado", que explicó la existencia de un sistema opresivo de dominio masculino construido sobre las mujeres en el ámbito privado, replicado en el ámbito público, donde el varón es la norma. Dada esta realidad, el feminismo proclamó la necesidad de superar la dicotomía público-privado e incorporar el ámbito privado al análisis de la política internacional ya que, como sostiene Enloe, “(...) se trata de demostrar cómo los Estados dependen de construcciones específicas en el ámbito privado para poder mantener particulares relaciones en el área pública e internacional". Es por ello que la autora adaptaba la frase de Beauvoir al campo de las Relaciones Internacionales, añadiendo "lo político es personal e internacional"5 (Locher, 1989, pp. 11-12).

Por otro lado, en la década de los ochenta aparece una nueva categoría analítica: el género. Ésta presentó una visión innovadora que entiende la importancia de alcanzar la esencia de las "identidades sexuales diferenciadas", reemplazando la categoría de mujer y de sexo (en un sentido biológico-positivista utilizado por otras corrientes). La categoría de género corre así el eje de atención hacia "la construcción social de la identidad sexual". El término fue utilizado por primera vez por Robert Stoller, sin embargo, la construcción teórica se le atribuye a la socióloga Ann Oaxley que, en 1972, definió al género como el abordaje que distingue lo masculino de lo femenino a partir de lo cultural, y lo diferencia del sexo, ligado a lo biológico. Esta mirada sería reformulada posteriormente por Gayle Rubin a través del concepto "sistema sexo/ género" (Aguilar García, 2008, pp. 3-5; Offen, 2006).

El objetivo de esta categoría es extender el estudio más allá de la mujer y centrar el foco de atención en la situación de interrelación de los sexos como un todo. En particular, se refiere a la histórica construcción de privilegio masculino frente a la desvalorización y marginación femenina y sus efectos negativos (Peterson, 2004, p. 39). Esta categoría presenta una flexibilidad, que permite su adaptación a diferentes realidades culturales, sociales y políticas en múltiples espacios y tiempo; en otras palabras, permite interpretar como sería "lo femenino" y "lo masculino" tanto en México a fines del siglo XIX, como en la China actual (Locher, 1998, p. 10). Además, la incorporación de la categoría de género en el análisis también permite enfocarnos en lo que sucede con las mujeres al interior de organismos internacionales o frente a acontecimientos

\footnotetext{
5 Traducción de la autora.
} 
mundiales que les atraviesan y, en consecuencia, promover políticas de acción. Sin embargo, es un concepto que presenta distintas interpretaciones que provocan debates y aparición de posiciones diversas en el interior del feminismo.

En referencia a las diversas categorizaciones sobre el modo de abordaje de las corrientes feministas dentro de las Relaciones Internacionales ${ }^{6}$ en este trabajo escogimos la evolución teórica desde un punto de vista cronológico por considerar que resulta más asequible, siendo la pionera la perspectiva liberal. Esta hizo visible la existencia de una estructura de pensamiento androcéntrica determinante que rige los espacios de poder internacionales. Por primera vez en la disciplina se develó la preexistencia de esta estructura hegemónica masculina, objetada a partir de silenciar la participación histórica de las mujeres. En otras palabras, se hizo manifiesto uno de los elementos característicos del pensamiento dominante masculino: la "invisibilidad de las mujeres" y, en confrontación a esta situación, se resignificó la presencia femenina dentro del campo de las Relaciones Internacionales.

El feminismo liberal contribuyó con el cuerpo teórico al incorporar la atención sobre el desempeño femenino en roles subordinados y marginados dentro de la política internacional, traduciéndolo en su aspecto militante, en la exigencia de una mayor participación femenina. Sin embargo, su aporte quedó limitado a la defensa de las mujeres construida sólo sobre una igualdad meramente cuantitativa, que mantuvo el patrón hegemónico masculino sin alcanzar a someterlo a una evaluación crítica. El enfoque epistémico-metodológico estuvo falto de alcanzar una consistencia que le permitiera superar la bifurcación de los ámbitos público y privado en el análisis internacional. Actualmente esta corriente se encuentra integrada, por ello carece de representantes académicas que se identifiquen en exclusividad con ella. (Locher, 1998, pp. 5- 6; Peterson, 2004, p. 38; Steans, 2006, pp. 12-13).

En una segunda instancia, emergió el standpoint o punto de vista feminista, que colocó el énfasis en la diferenciación de los sexos. Al igual que las feministas liberales, centró a las mujeres dentro de las Relaciones Internacionales, pero a diferencia de éstas fue más allá, cuestionando las estructuras y conceptos hegemónicos dentro de las Relaciones Internacionales. Para ello focalizó sus críticas en el realismo y el neorrealismo. Entre sus representantes más reconocidas se encuentra la especialista norteamericana J. Ann Tickner, que entiende que en la interpretación de los clásicos de la disciplina, el actor unificado exclusivo en el ámbito internacional ha sido el Estado con características "innatas" de racionalidad, y donde los "evidentes" intereses estatales en realidad representarían a aquellos que ocupan la escena pública, es decir a los varones, por tanto infiere que "no representaría ni epistemológicamente ni políticamente a las mujeres” (Steans, 2006, p. 14). Desde aquí surge su propuesta de revisionismo de las Relaciones Internacionales desde una perspectiva femenina.

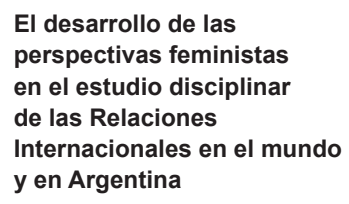

El desarrollo de las perspectivas feministas en el estudio disciplinar de las Relaciones Internacionales en el mundo y en Argentina

Mariel R. Lucero

\footnotetext{
6 Al respecto ver Wibben.
} 
Dossier América Latina: género y política
Sin embargo, sus detractores sostienen que este revisionismo femenino es excluyente, ya que presenta a "la mujer" desde un concepto universal; en otras palabras, esta corriente refleja la concepción de "una mujer blanca, anglosajona y de clase media". Los detractores entienden que la categoría "mujer" se encuentra atravesada por la geografía, por la época, por la etnia, entre otras variables. De hecho, en la perspectiva de Tickner la categoría "mujer" resulta ahistórica. A pesar de las limitaciones de los planteos de este standpoint la propuesta termina siendo válida en términos de cuestionamiento de las estructuras impuestas y como generadora de debate y pensamiento (Steans, 2006, p. 15).

En una tercera etapa, aparece el feminismo crítico, cuestionando también las estructuras, pero aquí las principales influencias teóricas provienen de la escuela marxista, en particular neo-gramsciana, que centra el análisis en las relaciones sociales de dominación. Al respecto, entiende que estas se construyen sobre "(...) la privatización del poder laboral productivo y reproductivo de las mujeres, el control de la sexualidad de las mujeres y la subordinación de las mujeres a la autoridad masculina a través de las instituciones patriarcales". Una de sus exponentes más prolíficas, Sandra Whitworth, entiende que el rol jugado por las ideas y las prácticas sociales, instituciones y organizaciones internacionales construyen, reconstruyen y reproducen esas formas de relacionamiento de carácter patriarcal. En este abordaje, la especialista sostiene que la atención debe centrarse en la categoría de género, y no exclusivamente en las mujeres, analizando el relacionamiento entre hombres y mujeres que incluye el poder que manifiesta la histórica discriminación y marginación femenina en diversos contextos (Steans, 2006, p. 15). Esta corriente, en particular, contempla no sólo la teorización, sino la práctica política, el proyecto emancipatorio como parte esencial de su actividad. Entre otras representantes también podemos ubicar a Jill Steans y Maxine Molyneaux.

Luego aparecería el feminismo posestructuralista. Esta corriente presenta una posición radicalizada, que cuestiona permanentemente el concepto de verdad, de lo único y de la identidad, oponiéndose a una visión unificada, incluso rechaza las categorías de mujer, varón, sexo y género. Entiende que, en la historia de la ciencia, la "verdad" ha generado la "otredad", la marginalidad de aquellos conocimientos que no se correspondían con el paradigma adoptado, lo cual permitió darle mayor centralidad al paradigma hegemónico. La radicalización de esta corriente se encuentra en la interpretación de la teorización como una forma de dominación donde los teóricos comprenden y se apropian del objeto de conocimiento marginando cualquier "otra" interpretación. En este sentido, el objetivo de esta corriente es develar. Para ello identifica los supuestos subyacentes en las teorías ya existentes y en los intentos por construir un metarrelato. El énfasis se centra en el "otro", lo revaloriza, siendo ésta su base para establecer el debate abierto y pluralidad, que incluye los discursos marginalizados e invisibilizados del "centro teórico" (Steans, 2006, pp. 16-17). Una de las referentes que se adhiere a este enfoque es Charlotte Hooper. 
Finalmente, surge el feminismo poscolonial que es la última corriente que incorpora al debate la dificultad para unificar la definición de mujer y destaca la heterogeneidad del colectivo "mujeres", colocando el énfasis en las diferencias de raza, etnia, clases y opresión sexual. En el análisis, conjuga distintas matrices de dominación como el patriarcado, el colonialismo y el racismo, sólo para mencionar las más difundidas. Uno de los conceptos centrales en torno a esta perspectiva es la "otredad" y el poder asimétrico que cruza las diferencias entre hombres y mujeres, y dentro del propio grupo de mujeres. Entre sus principales exponentes se encuentran Chandra Mohanty, Gayatri Spivak y Kumari Jayawardena (Steans, 2006, pp. 18-19).

En síntesis, la introducción de las perspectivas feministas en las Relaciones Internacionales descarta toda argumentación sobre la asexualidad o neutralidad sexual en la política internacional (Enloe, 1989), y la incorporación de los conceptos de patriarcado y género condujeron a un replanteo sobre las estructuras, conexiones y funcionalidad en la política internacional. En este sentido, presenta la necesidad de superar la dicotomía entre política exterior e interna, centrando su foco en las relaciones de dominación en los niveles privado/doméstico que se reproducen a nivel nacional e internacional. En general, encontramos muchas representantes del feminismo que difícilmente puedan encuadrarse dentro de una corriente en particular, como es el caso de Christine Sylvester, Marysia Zalewski y V. Spike Peterson, que seleccionan algunos conceptos particulares de cada una de ellas, construyendo su propio análisis e interpretación de la realidad.

\section{La perspectiva feminista en Argentina}

En general, el desarrollo de la disciplina de las Relaciones Internacionales en América Latina es muy reciente, en esto se destacan los aportes realizados desde los enfoques dependentistas. En Argentina en particular, la disciplina presentó su aporte principal asociado a la política exterior, teniendo su período de mayor crecimiento durante la consolidación democrática en los ochenta y noventa. En este punto es necesario aclarar que existe un importante desarrollo en las Ciencias Políticas, pero esto aún no se ve reflejado en las Relaciones Internacionales.

Según un estudio realizado en el año 2008 por los especialistas Federico Merkel y Florencia Montal sobre el campo de las Relaciones Internacionales en Argentina, el relevamiento sobre un grupo de universidades donde se dicta la carrera permitió inferir que el espacio curricular dedicado al ámbito teórico era reducido. En promedio, se dicta un curso de aproximadamente un año donde el mismo muchas veces se encuentra invadido por contenidos propios de la política exterior. En este sentido, los enfoques teóricos de las Relaciones Internacionales se presentan muy escasos en proporción al tiempo dedicado. En referencia a los enfoques escogidos en los programas estudiados, las
El desarrollo de las perspectivas feministas en el estudio disciplinar de las Relaciones Internacionales en el mundo y en Argentina

Mariel R. Lucero 
Dossier América Latina: género y política orientaciones teóricas más abordadas son las perspectivas realistas y liberales. Por otra parte, dentro de la corriente reflectivista, el enfoque constructivista se estudia, sin embargo, el posmodernismo y el feminismo en algunas instituciones no aparecen abordados (Merkel y Montal, 2008, pp. 5-7).

Sin duda, la inexistencia de los enfoques feministas desfavorece el surgimiento y divulgación de esta perspectiva, como también la presencia de estudios que estudien a las mujeres en las Relaciones Internacionales. Esto puede observarse en las estadísticas presentadas por TRIP del 2014 para Argentina donde no aparecen especialistas orientados u orientadas hacia esta área. $\mathrm{Al}$ respecto existen trabajos que estudian a las mujeres sin embargo carecen de un enfoque feminista de las Relaciones Internacionales.

El impacto en los últimos años de los fenómenos como el \#niunamenos o \#miracomonosponemos, sumados a otros procesos como la "marea verde", asociada a la lucha por la despenalización del aborto, renovaron el movimiento feminista en Argentina y, por ende, el interés favoreció la producción de estudios asociados a las mujeres, incluso en las Relaciones Internacionales. Esto generó la creación en 2018 del primer centro dedicado específicamente a los estudios de género en Relaciones Internacionales, el Centro de Estudios en Género(s) y Relaciones Internacionales (CEGRI), perteneciente al Instituto de Relaciones Internacionales (IRI) de la Universidad Nacional de La Plata, dirigido por Dulce Daniela Cháves, y por otro lado, el Centro de Estudios de Relaciones Internacionales y Medio Ambiente (CERIMA) en la Facultad de Ciencias Políticas y Sociales de la Universidad Nacional de Cuyo, de un área específica que trabaja el tema de género a través de un proyecto sobre migración y género (MIGEN).

Por otro lado, en algunas conferencias o reuniones científicas a nivel local se han generado espacios como en el XII Congreso Nacional de Ciencia Política, organizado por la Sociedad Argentina Análisis Político (SAAP) en 2015, realizado en la ciudad de Mendoza el panel "Visibilizando las mujeres en las Relaciones Internacionales", o en el marco del IX Congreso Internacional del IRI en el año 2018 el taller dictado por el CEGRI “Género(s) y Relaciones Internacionales: un diálogo necesario para deconstruir nuestra mirada”. Finalmente, en el año 2019, las II Jornadas Cuyanas de Relaciones Internacionales un eje temático sobre mujeres y Relaciones Internacionales. Este panorama y la inclusión, aunque sea nominal hoy, en las agendas temáticas de muchos institutos permiten prever un buen augurio para el desarrollo de este enfoque en Argentina.

\section{Conclusiones}

Indudablemente, las perspectivas feministas en las Relaciones Internacionales presentan una alternativa dentro de los enfoques teóricos mayor de aquella visión estereotipada que sólo ve en ella a "un grupo de mujeres que intentan 
estudiar temas de mujeres". En esta corriente, integrada por diversos enfoques, el feminismo replantea desde lo ontológico, lo epistemológico y lo metodológico el marco de estudio de las Relaciones Internacionales, integrando al nivel de análisis internacional, estatal, e individual, y cuestionando la dicotomía público-privado.

Los estudios feministas han crecido en los últimos 40 años a nivel global, instalando el debate, creando mesas y espacios dentro de asociaciones propias de la disciplina, promoviendo la difusión y producción, e incluso generando la reestructuración de la visión de las Relaciones Internacionales. Tal es así, que surge dentro de la disciplina una corriente de "nuevas masculinidades", siendo algunos y algunas de sus referentes como Terrell Carver o Molly Cochran, entre muchos otros y otras. Sin embargo, esto aún no ha tenido un impulso suficiente para generar un espacio dentro del cuerpo de los estudios teóricos de abordaje de la disciplina en Latinoamérica.

Más allá de la juventud de esta disciplina a nivel regional, y en particular en Argentina, que recién aparece en los años setenta, encuentra en la formación de sus profesionales un fuerte sesgo del paradigma realista que condiciona con la mirada patriarcal el análisis de la política mundial. Sin embargo, existen distintos acontecimientos y fenómenos que permiten prever un pronto florecimiento del enfoque feminista en Relaciones Internacionales en el país. Por un lado, los antecedentes asociados a un histórico desarrollo del movimiento feminista en Argentina y su política de derechos humanos con una gran cantidad de derechos sociales adquiridos; por otro lado, el actual contexto nacional y mundial asociado a la movilización y reclamos de las mujeres frente a la violencia de género, los abusos de poder y la defensa por la despenalización del aborto, sumado a un cupo prevaleciente femenino de estudiantes dentro de esta carrera permiten confirmar esta proyección.

\section{Referencias bibliográficas}

Aguilar García, T. (2008). Feminismo posmoderno: D. J. Haraway y S. Harding. Eidos: Revista de filosofía de la Universidad del Norte, pp. 222-232.

Enloe, C. (1989). Banana, Beaches \& Bases. Making Feminist Sense of International Politics. London: Pandora Press.

Jarvis, D. (2000). International Relations and the Challenge of Postmodernism. Defending the Discipline. California: University of South California Press.

Locher, B. (1998). Las relaciones desde la perspectiva de los sexos. Nueva Sociedad, no. 158 , (Noviembre/Diciembre), pp. 40-65.

Merkel, F. y Montal, F. (2008). En busca de una comunidad: el campo de las Relaciones Internacionales en la Argentina, ponencia presentada en el seminario "El status de las Relaciones Internacionales en Argentina", México (DF), 14 al 16 de octubre, organizado por ITAM y la Universidad de los Andes.
El desarrollo de las perspectivas feministas en el estudio disciplinar de las Relaciones Internacionales en el mundo y en Argentina

Mariel R. Lucero 
Dossier América Latina: género y política
Offen, K. (2006). El "gender", ¿es un invento americano?. Clio. Histoire, femme et societes. Recuperado 13.03.2019 de https://journals.openedition.org/clio/4702

Peterson, V. (2004). Feminist Theories Within, Invisible To, and Beyond IR. The Brown Journal of World Affairs, vol. 10, no. 2, pp. 35-46.

Sodupe, K. (2003). La teoría de las Relaciones Internacionales a comienzos del siglo XX. Bilbao: Universidad del País Vasco/Euskal Herriko Unibertsitatea.

Steans, J. (2006). Gender and International Relations. Issues, debate and future directions. Cambridge: Polity Press.

Sylvester, C. (1992). Feminist Theory and Gender Studies in International Relations. International Studies Notes, vol. 16/17, no. 3/1, pp. 32-38.

Sylvester, C. (2004). Feminist International Relations. An Unfinished Journey. Cambridge: Cambridge University Press.

Tickner, J. A. y True, J. (2018). A Century of International Relations Feminism: From World War I Women's Peace Pragmatism to the Women, Peace and Security Agenda. International Studies Quarterly, no. 62, pp. 221-233.

TRIP-Teaching, Research and International Policy (December 2018). TRIP Faculty Survey in Argentina. Recuperado 10.04.2019 de https://trip.wm.edu/charts/\#/questions/8

Wibben, A. (2004). Feminist International Relations: Old Debates and New Directions. The Brown Journal of World Affairs, vol. 10, no. 2, pp. 97-114. 